\title{
BMJ Open Perception of pharmacological prevention and subsequent non- adherence to medication in patients with ischaemic heart disease: a population-based cohort study
}

\author{
Christina Boesgaard Graversen (10, ,' Jan Brink Valentin (1) ,2 \\ Mogens Lytken Larsen, ${ }^{3}$ Sam Riahi, ${ }^{1,3}$ Teresa Holmberg, ${ }^{4}$ Line Zinckernagel, ${ }^{4}$ \\ Søren Paaske Johnsen ${ }^{2}$
}

To cite: Graversen $\mathrm{CB}$, Valentin JB, Larsen ML, et al. Perception of pharmacological prevention and subsequent non-adherence to medication in patients with ischaemic heart disease: a populationbased cohort study. BMJ Open 2022;12:e054362. doi:10.1136/ bmjopen-2021-054362

- Prepublication history and additional supplemental material for this paper are available online. To view these files, please visit the journal online (http://dx.doi.org/10.1136/ bmjopen-2021-054362).

Received 09 June 2021 Accepted 15 December 2021

Check for updates

(c) Author(s) (or their employer(s)) 2022. Re-use permitted under CC BY-NC. No commercial re-use. See rights and permissions. Published by BMJ.

For numbered affiliations see end of article.

Correspondence to Dr Christina Boesgaard Graversen; c.graversen@rn.dk

\section{ABSTRACT}

Objective A patient-focused approach is advocated to embody risk of non-adherence to medication and subsequent adverse clinical outcomes following ischaemic heart disease (IHD). This study aimed to explore how patient perceived information on pharmacological prevention was associated with subsequent nonadherence to medication (measured by non-initiation, non-implementation and non-persistence) in patients with incident IHD.

Design Cohort study.

Setting Denmark.

Participants Register-based cohort of 829 patients with incident IHD in 2013.

Measures Perception covered whether patients' experienced being adequately informed about their pharmacological prevention. Information on such was obtained from a survey and divided into 'Well informed', 'Moderately informed' and 'Poorly informed'. Information on baseline characteristics, and reimbursed prescriptions of medication (antiplatelets, statins, ACE-inhibitors/ angiotensin receptor blockers and $\beta$-blockers) during follow-up were obtained by linkage to nationwide public registers. Non-initiation and non-implementation of medication, measured as proportion of days covered, were analysed by Poisson regression. Non-persistence to medication, measured as risk of discontinuation, was analysed by multivariable Cox proportional hazard regression.

Primary and secondary outcome measures Nonimplementation and non-persistence to medication up to 365 days of follow-up were primary outcomes. Secondary outcomes included non-initiation as well as non-implementation and non-persistence to medication at 180 days of follow-up.

Results A dose-response association was in general found between perception of pharmacological prevention and risk of non-implementation and non-persistence. For example, the hazard of non-persistence to antiplatelets was 1.18 (95\% Cl 0.71 to 1.96) times higher for patients reporting 'Moderately informed' and $1.89(95 \% \mathrm{Cl} 1.10$ to 3.25) times higher for patients reporting 'Poorly informed',
Strengths and limitations of this study

- This study used patient-reported perception of pharmacological prevention from a register-based national survey.

- Non-adherence to medication was evaluated using a national prescription register and the categorisation followed the $\mathrm{ABC}$ taxonomy.

- The unique personal identification number enabled linkage to national register data sources at an individual level.

- There is no consensus on how to use patients' experience measures to evaluate adherence with pharmacological prevention.

compared with patients reporting 'Well informed of perception of pharmacological prevention' up to 365 days of follow-up.

Conclusion Lower levels of perception of pharmacological prevention were associated with subsequent nonimplementation and non-persistence to medication in patients with incident IHD.

\section{INTRODUCTION}

Adherence to cardiovascular medication for secondary prevention is essential to prevent recurrence of disease-related adverse outcomes including death in patients with ischaemic heart disease (IHD) ${ }^{1-3}$ However, studies have consistently found a timedependent progressive decline of adherence to secondary cardiovascular medication resulting in a large proportion of patients not receiving relevant treatment. ${ }^{45}$ The reasons for non-adherence are complex and include social/economic-related, patient-related, therapy-related, condition-related and healthcare team/system-related factors. ${ }^{6}$ 
In general, approximately $20 \%$ of the variance in adherence to medicine may be attributed to patients' perception of pharmacological prevention, including their expectations of treatment efficacy and perceived risk associated with their condition. ${ }^{7-10}$ Two studies observed beliefs about medication to be associated with self-reported non-adherence to medication in patients with IHD. ${ }^{11}{ }^{12}$ However, these studies are challenged by limitations including the use of self-reported data on adherence, use of cross-sectional study design with inclusion of prevalent rather than incident patients ${ }^{12}$ and a short follow-up period. ${ }^{11}$ Evaluating adherence measures is not trivial because of the risk of misclassification, which is lowered using a register-based approach compared with self-reported measures. Thus, there is a need to study how perception about pharmacological prevention is associated with non-adherence to medication using registerbased adherence measures with a longer follow-up period.

The present study aimed to investigate the association between patients' perception of pharmacological prevention and risk of non-adherence to register verified use of antiplatelets, statins, ACE inhibitors/angiotensin receptor blockers (ARBs) and $\beta$-blockers after hospital discharge among patients with incident IHD. Perception covered whether patients' experienced being adequately informed about their secondary pharmacological prevention including aspects regarding necessity, side-effects and regimen duration. It was hypothesised that lower levels of perception of pharmacological prevention increased risk of subsequent non-adherence to prescribed cardiovascular medication.

\section{METHODS}

\section{Study design}

A nationwide cohort study combining a baseline patientreported survey with register-based follow-up. Individuallevel record linkage with register data was done using the unique personal identification number provided to all Danish citizens. ${ }^{13}$

\section{Setting}

The Danish healthcare system is primarily financed through tax payment with equal access to healthcare. General reimbursement is given to Danish residents when buying medicine on prescription at the pharmacy, although some copayment by the patient is required. ${ }^{14}$

\section{Study population}

The study population consisted of a sample of 1742 patients with IHD (International Classification of Diseases (ICD)-10: I20-I25) diagnosed in 2013 and identified from the Danish National Patient Registry (NPR). ${ }^{15}$ The sample was part of a cohort consisting of 5000 patients with heart disease included in a national Danish survey. ${ }^{16}$ In short, the items used in the survey were selected after a thorough literature review and a qualitative explorative investigation. Then, it was pilot tested in individual and focus groups interviews and later evaluated by experts within the field of survey construction. The questionnaire was sent on 27 October 2014 and last day for answering was on 14 December 2014. Accordingly, patients received the questionnaire between 10 and 22 months after a diagnosis of incident IHD.

In the present study, patients were included if diagnosed with incident IHD in 2013 and no previous IHD diagnosis between 2008 and 2012, $\geq 35$ years of age, residents of Denmark and alive when the sample was established in October 2014. Figure 1 shows the exclusion criteria, which included non-responders, patients who stated in the survey that they were not diagnosed with a cardiac disease in 2013, missing answers on all three questionnaire items used as exposure (patients' perception of pharmacological prevention) in present study, or missing register information regarding sociodemographic variables.

\section{Patients' perception of pharmacological prevention}

Perception of pharmacological prevention was assessed by three questions in the survey that covered adequate information on secondary pharmacological prevention:

1. Do you feel informed about why you get medication for your heart disease?

2. Do you feel informed about possible side effects of medications for your heart disease?

3. Do you feel informed about how long you should take medication for your heart disease?

The participants were only allowed to use one response for each question (online supplemental table 1). The responses in each item were divided into three subgroups and graded the responses with points (Well informed (0 points); Moderately informed (1 point); Poorly informed, including 'do not know' responses (2 points)). Then, a composite measure was constructed by summarising the responses and categorised the patients according to their average score in the three items (score ranged from 0 to 6): Well informed (score $=0$ ); Moderately informed ( score $=1-3$ ); Poorly informed (score $=4-6$ ). This measure worked as definition of patients' perception of pharmacological prevention.

\section{Non-adherence to cardiovascular medication}

Definition of non-adherence followed the ABC taxonomy and ESPACOMP Medication Adherence Reporting Guideline (EMERGE) criteria, including non-initiation (does not initiate treatment), non-implementation (does not take medication as prescribed) and non-persistence (discontinues treatment). ${ }^{1718}$ The primary endpoints were non-implementation and non-persistence up to 365 days follow-up. Non-initiation as well as non-implementation and non-persistence up to 180 days follow-up were defined as secondary endpoints. Non-initiation was categorised as failure to pick up a prescription within 180 days following hospital discharge. Non-implementation was evaluated with proportion of days covered (PDC) and non-persistence to risk of drug discontinuation, that 
1742 Patients diagnosed with ischemic heart disease in 2013 and eligible for participation in survey

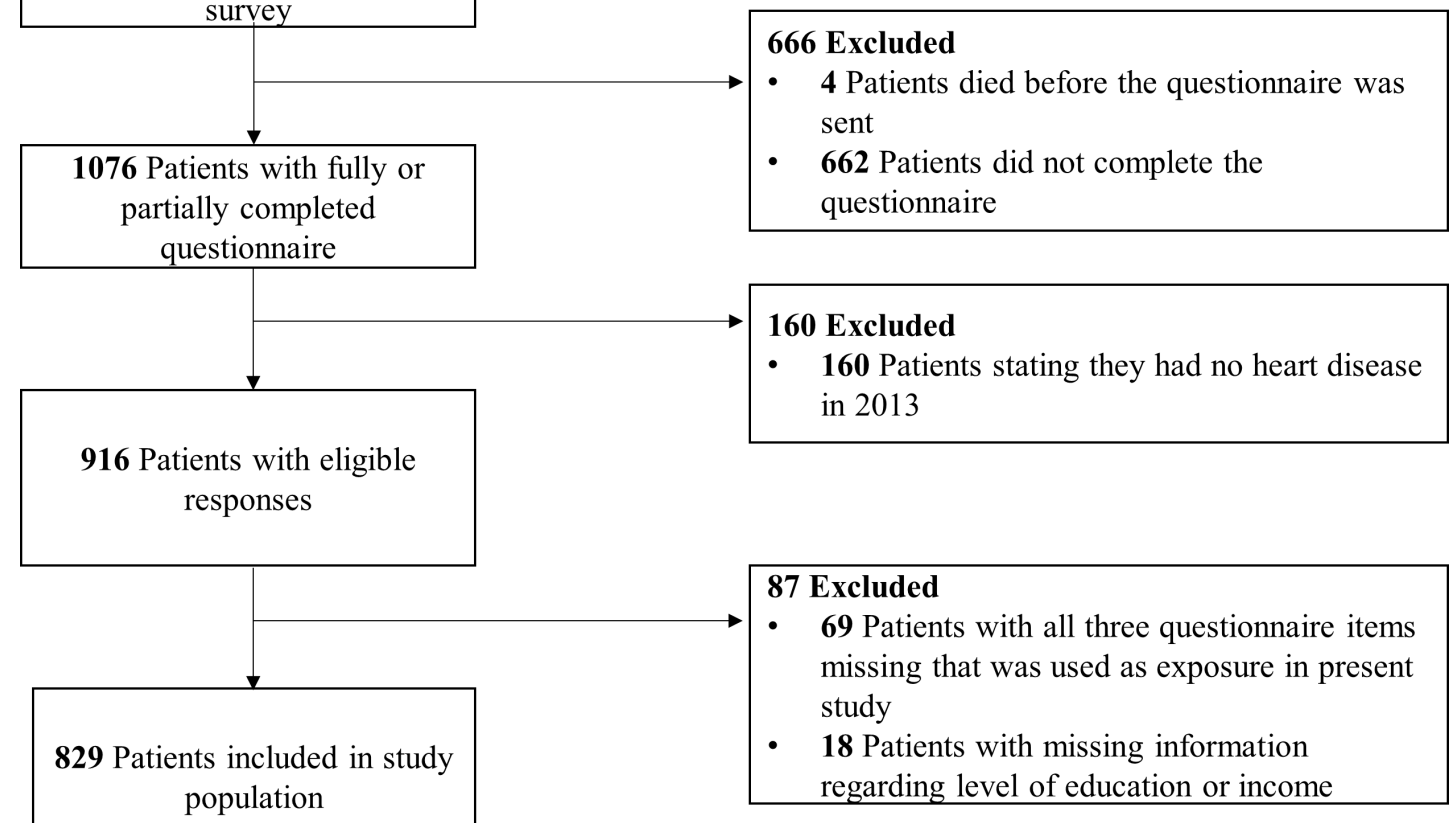

Figure 1 Study population.

was defined as failure to pick up a new prescription for preventive medication with a grace period of 90 days after estimated date of expiry of a reimbursed prescription.

Data on reimbursed medication were provided by the Danish National Prescription Registry (DNPR), which captures all prescriptions filled by Danish residents at pharmacies since $1995 .{ }^{19}$

Secondary preventive IHD medications included antiplatelets, statins, $\beta$-blockers, ACE-inhibitors/ARBs Anatomical Therapeutic Chemical Classification System (ATC)-codes available in online supplemental table 2). A daily dose of one tablet was pragmatically chosen irrespectively of drug class. As the DNPR do not capture drugs dispensed during hospitalisation, patients were hypothesised to receive medication in the hospital if having a readmission lasting longer than 7 days to reduce risk of misclassification of events.

\section{Potential confounding factors}

The choice of confounding variables was assessed using directed acyclic graph depicting knowledge from reviews of existing studies and assumptions about the possible causal interrelationships between the exposure and outcome (online supplemental figure 1). The confounding variables included age at diagnosis, sex, cohabiting status, ethnicity, occupational status, household income, educational level, somatic comorbidity and level of supporting relatives.

Information regarding age at diagnosis, sex, cohabiting status (living alone or cohabiting) and ethnic background (Danish, immigrant, descendant) was provided from The Danish Civil Registration System. ${ }^{13}$ Information regarding occupational status (employed, unemployed, retired/outside labour force) was provided from the Register-based Labour Force Statistics. ${ }^{20}$

The equalised disposable household income (considering household income and family size) from the Income Statistic Register was used to calculate the average income for a 5-year period (2008-2012) prior to diagnosis in order to capture yearly income fluctuation. ${ }^{21}$ Income was divided into tertiles.

The highest attained educational level was obtained using the Population Education Register from the year prior to IHD diagnosis and divided into three subgroups according to the International Standard Classification of Education (low ( $<10$ years of education), middle (10-12 years of education), high $(>12$ years of education) $) .{ }^{22} 23$ Information regarding somatic comorbidities 10 years prior to IHD diagnosis was defined from the Charlson Comorbidity Index (none (score $=0$ ), moderate (score $=1$ 2), high (score $>2)$ ) and provided from the NPR.

Level of supporting relatives was obtained with a question in the survey asking if patients felt they had relatives they could rely on (Well informed; Moderately informed; Poorly informed/not at all).

Other covariates were included to describe the study population, but these were not used to control for confounding (Procedures: coronary angiography, percutaneous coronary intervention, coronary artery bypass grafting; medication before hospitalisation; information regarding individual consultation about medication in 
which patients were asked if they in connection to their disease were offered (and received) an individual consultation about medication.) (Procedure-codes and ATCcodes available in online supplemental table 2).

\section{Statistical methods}

The McDonalds omega was calculated to assess item consistency for the summed measure of patient perception; a value $\geq 0.70$ was considered to represent sufficient consistency. ${ }^{24}$

The association between patients' perception of pharmacological prevention and risk of non-initiation of medication was evaluated using Poisson regression with robust error variance.

Two regression measures assessed the association between patients' perception of pharmacological prevention and risk of non-implementation and non-persistence to medication after initial initiation. Each secondary preventive medication group was analysed separately. First, non-implementation in adherence to medication was evaluated using PDC and analysed by Poisson regression with introduction of robust error variance. Implementation of medication was grouped into good (defined as $\geq 80 \%$ adherence to medication) or poor $(<80 \%$ adherence to medication). Patients were followed from time of initiation until a censoring event (emigration, death), or end of follow-up at 180 or 365 days. Results were reported as relative risk (RR) with $95 \% \mathrm{CI}$.

Second, non-persistence was evaluated using multivariable Cox proportional hazard regression model to examine risk of discontinuation among those initiating secondary preventive medication. Patients were followed from time of initiation until time of endpoint (drug discontinuation), a censoring event (emigration, death) or end of follow-up at 180 or 365 days. Results were reported as HR with 95\% CI. The proportional hazard models were checked using graphical means performed by log-log plots and visually analysed to fulfil the assumption.

To account for missing data in the patient-reported measures $(<10 \%)$, we used multiple imputation chained equations and imputed 10 sets under the assumption of data being missing at random. The imputation model employed the proportional odds logistic regression model for each of the variables with missing data, and included the variables presented in table 1 as well as relevant outcome and exposure variables. Several supplementary analyses were undertaken. First, a non-response analysis was undertaken and an analysis using the three survey items separately as exposures was performed. Then, data were stratified according to sex to evaluate potential effect modification. Additionally, a sensitivity analysis was performed that included 'medication before hospital admission' as covariate and an analysis using an alternative grace period of 60 days. Further, the association between perception of pharmacological prevention and risk of non-adherence to dispensed combination of one, two (antiplatelets and statins), three (additional
ACE-inhibitor/ARBs or $\beta$-blockers) or all four drug classes was investigated.

All analyses were performed using Stata Software (V.16.1; Stata Corp.).

\section{Patient and public involvement}

Patients and caregivers were involved in the development of the questionnaire.

\section{RESULTS}

\section{Respondent baseline characteristics}

The study population comprised 829 patients with incident IHD, corresponding to $47.6 \%$ of the initial population eligible for participation in the survey (figure 1). Table 1 presents sociodemographic-related, procedurerelated and health-related baseline characteristics of the respondents. Perception of pharmacological prevention was reported 'Well informed' by $25.5 \%$ patients, 'Moderately informed' by $45.8 \%$ patients and 'Poorly informed' by $28.7 \%$ patients.

A larger proportion of those patients reporting Poorly informed were older, better educated, unmarried/single living, retired and were less likely to report 'a high level' of perception of supportive relatives or having received individual consultation in medication in relation to cardiac rehabilitation or at the general practitioner. The responses of the three individual survey items are presented in online supplemental table 2.

A total of $345(41.6 \%)$ patients were on antiplatelets, $400(48.3 \%)$ patients were on statins, $226(48.8 \%)$ patients were on ACE-inhibitors/ARBs and 252 (37.1\%) patients were on $\beta$-blockers prior to incident hospitalisation for IHD. Finally, a moderate internal consistency was found for the summed measure of patient perception (McDonalds omega $=0.67$ ).

\section{Non-respondent baseline characteristics}

Non-respondents were classified as patients who either did not complete the questionnaire $(n=662)$ or had all three questionnaire items missing that was used as exposure in present study $(n=69)$. Non-respondents were slightly younger and more often women, non-Danish ethnic origin, lower educated, unmarried/single living (online supplemental table 3). Compared with respondents, a higher proportion of non-respondents did not initiate antiplatelets or statins (online supplemental table 4).

\section{Proportion of non-adherence to medication}

The number of patients being assessed for non-adherence to medication in the four study groups included: 829 patients regarding antiplatelets; 829 patients regarding statins; 463 patients regarding ACEI/ARBs; and 680 patients regarding $\beta$-blockers.

The number of patients not initiating medication was $77(9.3 \%)$ for antiplatelets, $65(7.7 \%)$ for statins, 61 
Table 1 Baseline characteristics according to patients' perception of pharmacological prevention

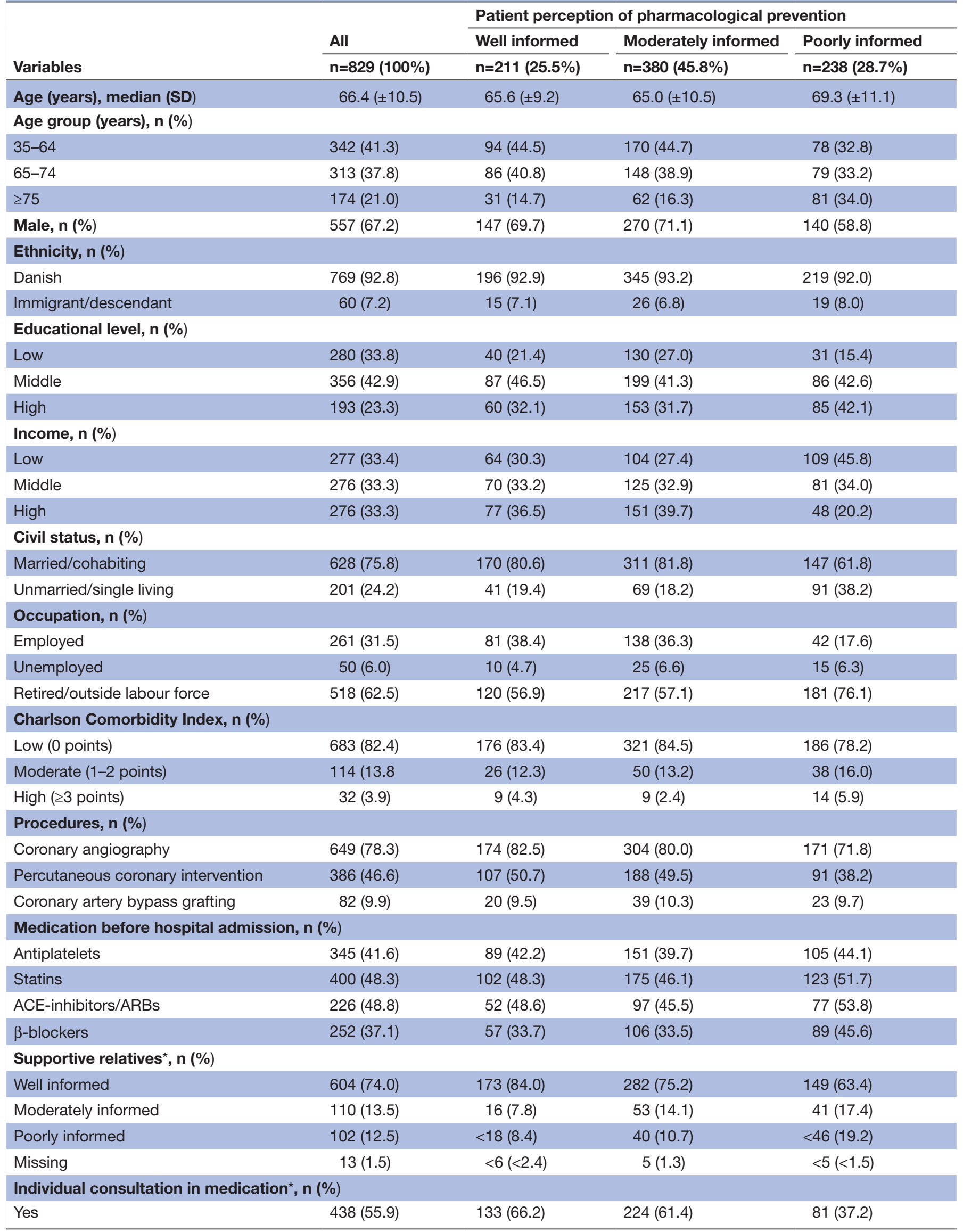


Table 1 Continued

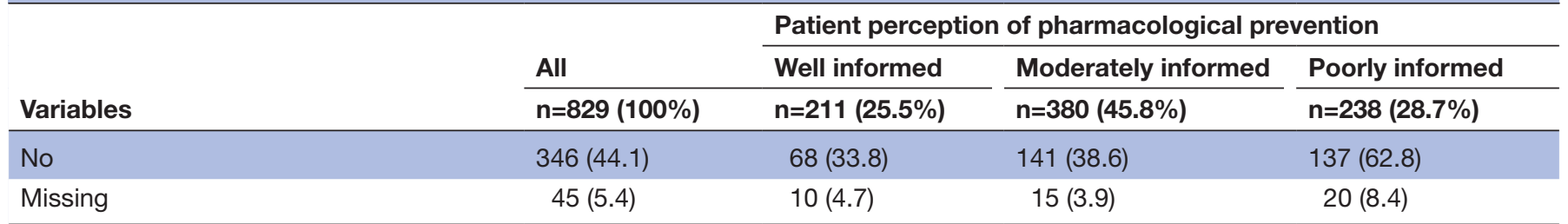

*Patient-reported experiences obtained from survey.

ARBs, angiotensin receptor blockers.

(13.2\%) for ACE-inhibitors/ARBs and 30 (4.4\%) for $\beta$-blockers (figure 2).

The highest risk was observed within the first 180 days of follow-up in which approximately $10 \%-15 \%$ of the patients had poor implementation of antiplatelets, statins or $\beta$-blockers, and approximately $22 \%$ of the patients were non-adherent to ACE-inhibitors/ARBs.

Between $3.2 \%$ and $8.0 \%$ of the patients were nonpersistent to antiplatelets, statins or $\beta$-blockers and approximately $20 \%$ of the patients were non-persistent to ACE-inhibitors/ARBs up to 180 days of follow-up. Between $15.2 \%$ and $30.6 \%$ of patients were non-persistent to their medical treatment up to 365 days of follow-up (figure 2).

\section{Association between patients' perception of pharmacological prevention and risk of non-initiation of medication}

The association between patients' perception about pharmacological prevention and subsequent non-initiation was not uniform and neither of the results reach statistical significance (figure 3). Overall, there was a tendency that patients' reporting Poorly informed of perceived information about pharmacological prevention had risk of subsequent non-initiation of antiplatelets (adjusted RR: $1.55,95 \%$ CI 0.81 to 2.96), statins (adjusted RR: 1.34,
95\% CI 0.64 to 2.80) and ACE-inhibitors/ARBs (adjusted RR: 1.09 , 95\% CI 0.49 to 2.41). Contrary, an opposite tendency was observed $\beta$-blockers (adjusted RR: 0.67, $95 \%$ CI 0.23 to 1.93 ). Thus, the evaluation of risks was inconclusive regarding perception of pharmacological prevention and subsequent non-initiation.

Association between patients' perception of pharmacological prevention and risk of non-implementation and nonpersistence to medication

Among those initiating medication, there was a general tendency that patients reporting lower levels of perception of pharmacological prevention had a gradually increase in risk of non-implementation and non-persistence across all groups of medication therapy, compared with those reporting Well informed (figure 4). These associations were observed both at 180 and 365 days of follow-up, irrespectively of medication class.

For example, up to 365 days of follow-up, patients reporting Poorly informed had a higher risk of nonimplementation of antiplatelets (adjusted RR: 1.65, 95\% CI 0.95 to 2.93 ), statins (adjusted RR: $1.70,95 \%$ CI 1.05 to 2.75), ACE-inhibitor/ARBs (adjusted RR: 1.76, 95\% CI 1.00 to 3.12 ) and $\beta$-blockers (RR: $1.26,95 \% \mathrm{CI}$

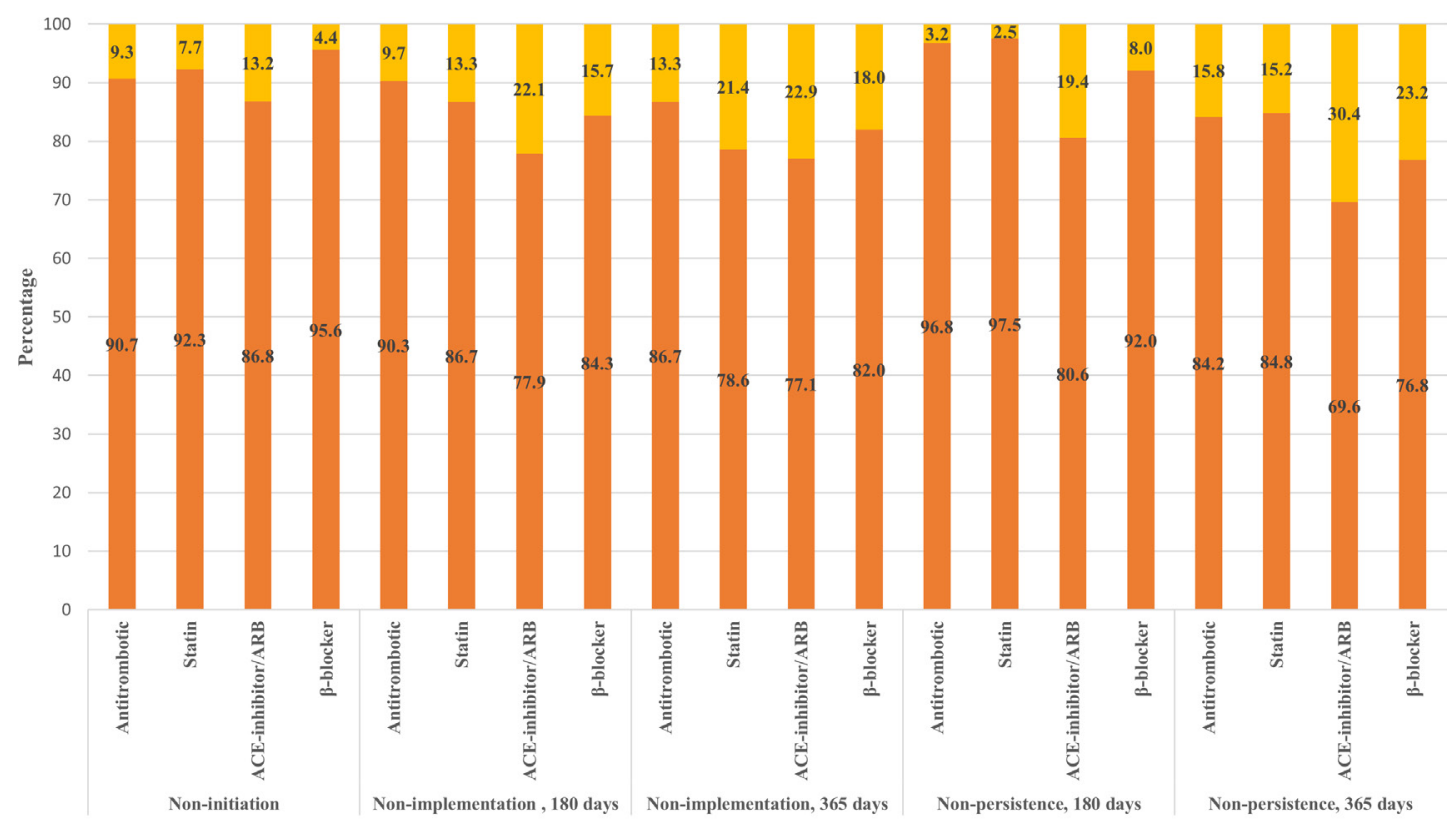

m Adherent $=$ Non-adherent

Figure 2 Proportion of patients being non-adherent to medication. ARB, angiotensin II receptor blockers. 


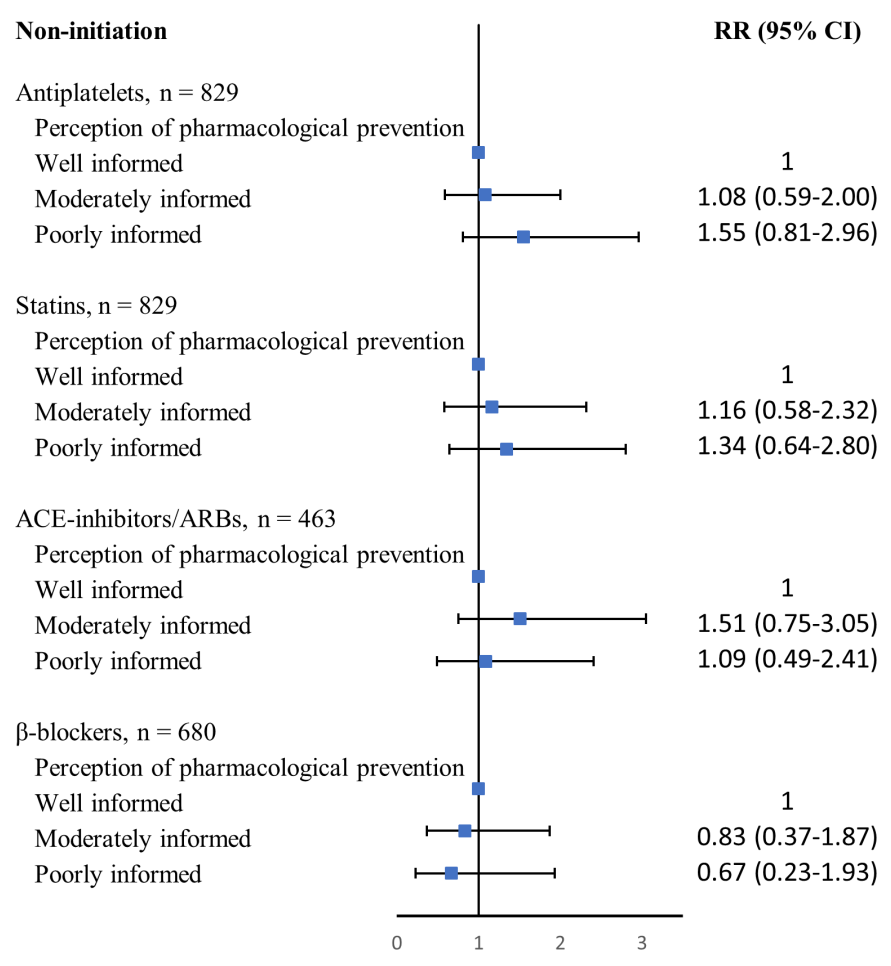

Figure 3 Adjusted association between patients' perception of pharmacological prevention and risk of non-initiation to medication. Adjusted for age, sex, ethnicity, income, education, civil status, occupation, comorbidity and supportive relatives. ARB, angiotensin II receptor blockers; $\mathrm{RR}$, relative risk.

0.77 to 2.07) compared with patients reporting Well informed of perception of pharmacological prevention.

Same tendencies were observed in the analyses estimating risk of non-persistence. Compared with patients reporting Well informed, patients reporting Poorly informed were more likely to discontinue antiplatelets (adjusted HR: 6.54, 95\% CI 1.38 to 29.18) up to 180 days of follow-up. Again, the tendencies were found in the other drug classes. Up to 365 days of follow-up, patients' reporting Poorly informed of perception had subsequent higher risk of non-persistence to antiplatelets (adjusted HR: $1.89,95 \%$ CI 1.10 to 3.25 ), statins (adjusted HR: 2.44, 95\% CI 1.39 to 4.29), ACE-inhibitors/ARBs (adjusted HR: $2.05,95 \%$ CI 1.06 to 3.95 ) and $\beta$-blockers (adjusted HR: $1.80,95 \%$ CI 1.03 to 3.12 ) compared with patients reporting Well informed of perception of pharmacological prevention.

Online supplemental table 5 presents the crude results evaluating the association between patient perception of pharmacological prevention and risk of non-adherence to medication.

\section{Supplementary analyses}

Results from the stratified analysis according to sex did not find indication of any differences compared with the main results (online supplemental table S6.1-S6.2).

Additional adjustment for medication before hospital admission did not change the results in a clinically significant matter when compared with the main analysis (results not shown). Further, the results were analysed using a shorter grace period of 60 days, but again, the results remained almost unchanged (results not shown).

The analyses were also performed using the three items as individual exposures but no major statistically or clinically important implications compared with the summed item measure was found (online supplemental table S7.1-S7.4).

Finally, the association between perception of pharmacological prevention and risk of non-adherence to dispensed combination of one, two or three drug classes was investigated. The proportion of non-initiation was $13.6 \%$ for 2 drugs (antiplatelets and statins), $21.8 \%$ for 3 drugs (additional ACE-inhibitors/ARBs), 14.3\% for 3 drugs (additional $\beta$-blockers) and $11.9 \%$ for all four drugs (online supplemental table 8 ). Patients reporting Poorly informed had higher risk of being non-adherent, irrespectively of drug combination and adherence measure (online supplemental table 9).

\section{DISCUSSION}

In this nationwide cohort study, lower levels of perceived information about secondary cardiovascular pharmacological prevention were associated with risk of nonimplementation and non-persistence to medication, especially up to 365 days of follow-up. These results were in general observed regardless of medication class. The findings of non-initiation were, however, inconclusive.

\section{Patients' perception of pharmacological prevention}

Diverse conceptualisations and methodological tools have been used to identify factors associated with risk of non-adherence but challenges remain when it comes to fully understand and improve adherence to medication. ${ }^{25}$ Looking beyond patient risk factors (age, comorbidities, socioeconomic factors), a good patient-provider relationship and communication may contribute to a better patient experience thus making the patient more engaged in disease management.

There is no consensus on how to use patients' experiences measures to evaluate adherence of pharmacological prevention. Studies using 'the Beliefs in Medication Questionnaire-Specific' (consists of a ten-statement questionnaire) find patients' perceptions of necessity and concerns regarding medical treatment to be associated with adherence to medication in general chronic disease $^{26}$ as well as in IHD patients. ${ }^{11} 12$

The two existing studies on patients with IHD found that low perception of necessity and high perception of concern were associated with increased risk of nonadherence. However, the studies used self-reported adherence measure and had limitations regarding the study designs including short follow-up time, ${ }^{11}$ cross-sectional study design ${ }^{12}$ and risk of selection bias due to the inclusion of prevalent patients. ${ }^{12}$

Despite perceived information on medication was only measured by three items in the current study, a 

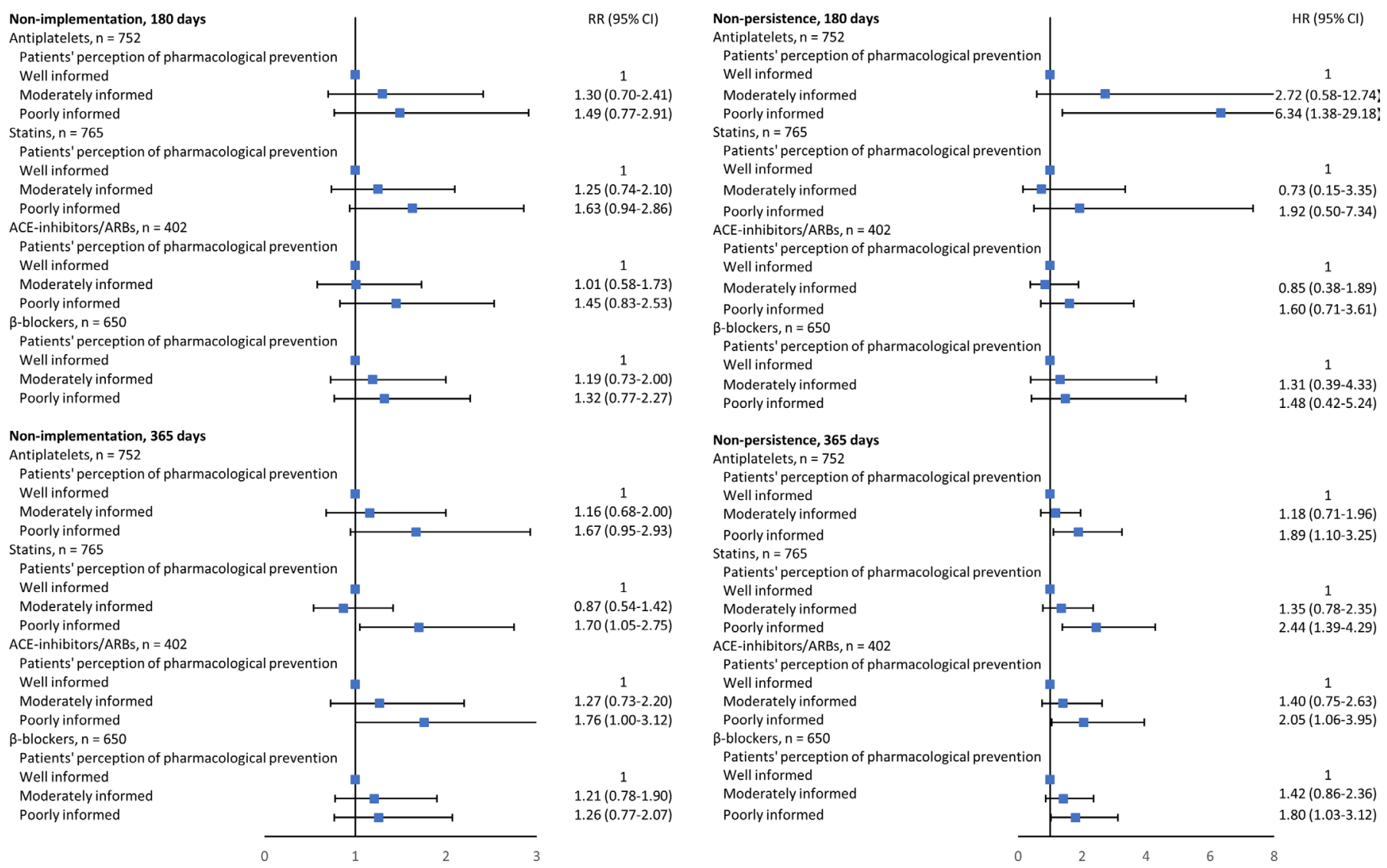

Figure 4 Adjusted association between patients' perception of pharmacological prevention and risk of non-implementation and non-persistence to medication. Adjusted for age, sex, ethnicity, income, education, civil status, occupation, comorbidity, and supportive relatives. ARB, angiotensin II receptor blockers; RR, relative risk.

dose-response association between patients' perception of pharmacological prevention and risk of nonimplementation and non-persistence to medication was in general observed. The strongest and most uniform results were observed regarding non-persistence to medication, compared with non-implementation to medication, up to 365 days of follow-up.

\section{Adherence measure and proportion of patients being non- adherent to medication}

Several methodological approaches have been proposed when estimating adherence to medication using pharmacy administrative databases. ${ }^{27}$ Without a single 'gold standard' approach, research suggests that adherence to medication should be evaluated by a combination of methods. ${ }^{28}{ }^{29}$ Despite this, abundant studies evaluate adherence to medication using a single measure, and only a limited number of studies have used multiple methodological approaches in cardiac patients. Two different methods (PDC (measure of implementation), risk of discontinuation (measure of persistence)) were applied to study aspects of patients' use of medication after having initiated treatment. Along with an analysis of non-initiation, it was thus possible to evaluate diverse aspects of non-implementation and non-persistence to medication in present study.
It was found that the proportion of patients not initiating therapy ranged from approximately 4.5\%-13\% depending on class of medication, which corresponds to earlier findings. ${ }^{30}$ Furthermore, the proportion of non-implementation and non-persistence to medication seemed to differ across drug class.

\section{Strengths and limitations}

This study was strengthened by its linkage of patients' perception of pharmacological with data from nationwide registers. The Danish registers have been validated for use in epidemiological research and they provide a comprehensive data material, which enables careful adjustment for confounding for each participant in present study. ${ }^{15}$ Further, non-adherence to medication followed the ABC taxonomy and was defined by a thorough approach using pharmacy register data of high quality. All approaches, alongside a range of sensitive analyses, showed almost similar results, which strengthened the study validity.

The present study also has some limitations that should be taken into consideration. The survey data were limited by item non-response. However, our data fulfilled the assumptions for multiple imputation as all item nonresponses were categorised as being missing at random and each item had less than $10 \%$ of the answers missing. The original questionnaire was validated for a broad 
patient experience, and we only used a limited selection of the questions which were considered to be specifically related to drug adherence. We cannot exclude the possibility that other questions may also have been related to drug adherence.

Theory find a McDonalds omega $\geq 0.70$ to equal a risk of $10 \%$ of misclassification if sufficient consistency is expected. Thus, a McDonalds omega $\geq 0.70$ is currently used as the standard cut-off measure. ${ }^{24}$ Given the nature of the questionnaire items, somewhat moderate to high consistency was expected and, although, a moderate consistency may represent failure to understand or perceive the questions, it may also simply express multidimensionality. Thus, with a McDonalds omega estimate of 0.67 , the risk of misclassification was estimated to be acceptable.

The survey had a moderate response proportion $(47.5 \%)$ with minor differences in baseline characteristics among respondents and non-respondents. A slight difference in initiation of medication was observed and it cannot be ruled out that this may have underestimated the proportion of patients not adherent to medication and thus affected the accuracy of the risk estimates.

The questions assessed both prior and present use of medication thereby reducing risk of poor recall due to delayed data collection. Prediction of the consequences of such delay can be difficult, but it seems most likely that a resulting misclassification would be non-differential rather than result in a systematic bias. Accordingly, it was believed that any change in patients' perception of pharmacological prevention would be minimal in the chosen time span, but this hypothesis has not been tested.

Compared with self-reported adherence to medicine, use of register data to measure adherence diminishes the risk of recall bias and any risk of loss to follow-up. However, the register was not created for scientific purposes and there might be a risk of incomplete data. For instance, the register only contained information regarding reimbursed prescriptions and did not evaluate if medication was taken as prescribed. However, this could be estimated when looking at the time span between reimbursement. Further, there was no information on non-reimbursed prescription, which made it impossible to differentiate between patients being primary non-adherent, and patients not prescribed cardiovascular medication. Also, there was no information on reasons for discontinuing the treatment, for example, contradiction/intolerance to a drug or adverse events.

Adherence to medication was most likely correlated within an individual and should be taken into consideration when interpreting the results. This occurred as all were patients prescribed antiplatelets and statins and most also with $\beta$-blockers. The online supplemental tables S8 and S9 evaluate the effect of the combination of drug classes. The first by presenting the number of patients being non-adherent to different drug combinations; the latter by analysing the association between perception of pharmacological prevention and risk of non-adherence to the different drug combination. No clinical major differences in the results were found when comparing to the main results presented in figures 3 and 4 .

In conclusion, this nationwide cohort study showed a dose-response association between lower levels of patients' perception of pharmacological prevention and risk of non-implementation and non-persistence to medication among patients with incident IHD. The association was with few exceptions observed irrespectively of medication class and most uniform up to 365 days of follow-up. These findings imply that improvement of patients' perception of pharmacological prevention may be an important tool to reduce the risk of non-implementation and non-persistence to cardiovascular medication and thus lowering the risk of adverse clinical outcomes and improving quality of life.

\section{Author affiliations}

${ }^{1}$ Department of Cardiology, Aalborg University Hospital, Aalborg, Denmark

${ }^{2}$ Danish Center for Clinical Health Services Research, Aalborg University, Aalborg, Denmark

${ }^{3}$ Department of Clinical Medicine, The Faculty of Medicine, Aalborg University, Aalborg, Denmark

${ }^{4}$ National Institute of Public Health, University of Southern Denmark, Copenhagen, Denmark

Acknowledgements A sincere appreciation to the patients completing the survey and all participants involved in the development of the questionnaire 'Living with heart disease'.

Contributors CBG, LZ and TH contributed to data acquisition. All authors contributed to study design and interpretation of data. CBG performed the statistical analysis and JBV contributed. CBG drafted the manuscript. SR, JBV, MLL, TH, LZ and SPJ critically revised the manuscript for finalisation. Final approval was given by all authors who also agreed to be accountable for every aspect of the work. CBG acts as overall responsible for the work of the study.

Funding This work was supported by the Danish Heart Foundation (grant number: 18-R123-A8283-22081).

Competing interests SPJ has provided consultant work for Bayer, Bristol-Myers Squibb, Pfizer and Sanofi and has participated in advisory board meeting for Bayer, Bristol-Myers Squibb and Pfizer. None of the other authors have conflicts of interest to declare.

Patient consent for publication Not applicable.

Ethics approval The study was approved by the University of Southern Denmark (no. 17/8592 (10.735)). According to Danish law, this type of study does not require further formal ethical approval. The letter sent to patients together with the survey explained the study aim, assuring them that participation was voluntary and that results would be anonymous. Participants provided informed consent for participation by returning the questionnaire.

Provenance and peer review Not commissioned; externally peer reviewed.

Data availability statement Data underlining this article will be shared upon reasonable request. Data were provided by a third party and are not publicly available.

Supplemental material This content has been supplied by the author(s). It has not been vetted by BMJ Publishing Group Limited (BMJ) and may not have been peer-reviewed. Any opinions or recommendations discussed are solely those of the author(s) and are not endorsed by BMJ. BMJ disclaims all liability and responsibility arising from any reliance placed on the content. Where the content includes any translated material, BMJ does not warrant the accuracy and reliability of the translations (including but not limited to local regulations, clinical guidelines, terminology, drug names and drug dosages), and is not responsible for any error and/or omissions arising from translation and adaptation or otherwise.

Open access This is an open access article distributed in accordance with the Creative Commons Attribution Non Commercial (CC BY-NC 4.0) license, which 
permits others to distribute, remix, adapt, build upon this work non-commercially, and license their derivative works on different terms, provided the original work is properly cited, appropriate credit is given, any changes made indicated, and the use is non-commercial. See: http://creativecommons.org/licenses/by-nc/4.0/.

\section{ORCID iDs}

Christina Boesgaard Graversen http://orcid.org/0000-0002-9199-6418

Jan Brink Valentin http://orcid.org/0000-0002-8205-7179

\section{REFERENCES}

1 Ibanez B, James S, Agewall S, et al. 2017 ESC Guidelines for the management of acute myocardial infarction in patients presenting with ST-segment elevation. Eur Heart J 2018;39:119-77.

2 Roffi M, Patrono C, Collet J-P, et al. 2015 ESC guidelines for the management of acute coronary syndromes in patients presenting without persistent ST-segment elevation: Task force for the management of acute coronary syndromes in patients presenting without persistent ST-segment elevation of the European Society of cardiology (ESC). Eur Heart J 2016;37:267-315.

3 Piepoli MF, Hoes AW, Agewall S, et al. 2016 European guidelines on cardiovascular disease prevention in clinical practice. Eur Heart $J$ 2016;37:2315-81.

4 Mathews R, Wang TY, Honeycutt E, et al. Persistence with secondary prevention medications after acute myocardial infarction: insights from the TRANSLATE-ACS study. Am Heart J 2015;170:62-9.

5 Naderi SH, Bestwick JP, Wald DS. Adherence to drugs that prevent cardiovascular disease: meta-analysis on 376,162 patients. Am J Med 2012;125:882-7.

6 World Health Organization. Long-term adherence to medication: evidence for action,, 2003. Available: https://www.who.int/chp/ knowledge/publications/adherence_full_report.pdf?ua=1 [Accessed 23 April 2020].

7 Gatti ME, Jacobson KL, Gazmararian JA, et al. Relationships between beliefs about medications and adherence. Am J Health Syst Pharm 2009;66:657-64.

8 Andersson Sundell K, Jönsson AK. Beliefs about medicines are strongly associated with medicine-use patterns among the general population. Int J Clin Pract 2016;70:277-85.

9 Horne R, Weinman J. Patients' beliefs about prescribed medicines and their role in adherence to treatment in chronic physical illness. $J$ Psychosom Res 1999;47:555-67.

10 Horwitz RI, Horwitz SM. Adherence to treatment and health outcomes. Arch Intern Med 1993;153:1863-8.

11 Allen LaPointe NM, Ou F-S, Calvert SB, et al. Association between patient beliefs and medication adherence following hospitalization for acute coronary syndrome. Am Heart J 2011;161:855-63.

12 Byrne M, Walsh J, Murphy AW. Secondary prevention of coronary heart disease: patient beliefs and health-related behaviour. $J$ Psychosom Res 2005;58:403-15.

13 Pedersen CB. The Danish civil registration system. Scand J Public Health 2011;39:22-5.
14 Danish Medicines Agency. General reimbursement of medicines, 2018. Available: https://laegemiddelstyrelsen.dk/en/reimbursement/ general-reimbursement/

15 Schmidt M, Schmidt SAJ, Sandegaard JL, et al. The Danish national patient registry: a review of content, data quality, and research potential. Clin Epidemiol 2015;7:449-90.

16 Zinckernagel L, Schneekloth N, Zwisler A-DO, et al. How to measure experiences of healthcare quality in Denmark among patients with heart disease? the development and psychometric evaluation of a patient-reported instrument. BMJ Open 2017;7:e016234.

17 Vrijens B, De Geest S, Hughes DA, et al. A new taxonomy for describing and defining adherence to medications. Br J Clin Pharmacol 2012;73:691-705.

18 De Geest S, Zullig LL, Dunbar-Jacob J, et al. ESPACOMP medication adherence reporting guideline (emerge). Ann Intern Med 2018;169:30-5.

19 Kildemoes HW, Sørensen HT, Hallas J. The Danish national prescription registry. Scand J Public Health 2011;39:38-41.

20 Petersson F, Baadsgaard M, Thygesen LC. Danish registers on personal labour market affiliation. Scand J Public Health 2011;39:95-8.

21 Baadsgaard M, Quitzau J. Danish registers on personal income and transfer payments. Scand J Public Health 2011;39:103-5.

22 Jensen VM, Rasmussen AW, W Ra. Danish education registers. Scand J Public Health 2011;39:91-4.

23 Eurostat Statistics Explained. International standard classification of education (ISCED). Available: https://ec.europa.eu/eurostat/ statistics-explained/index.php/International_Standard_Classification_ of_Education_\%28ISCED\%29

24 Nájera Catalán HE. Reliability, population classification and weighting in multidimensional poverty measurement: a Monte Carlo study. Soc Indic Res 2019;142:887-910.

25 Osterberg L, Blaschke T. Adherence to medication. N Engl J Med 2005;353:487-97.

26 Horne R, Chapman SCE, Parham R, et al. Understanding patients' adherence-related beliefs about medicines prescribed for longterm conditions: a meta-analytic review of the Necessity-Concerns framework. PLoS One 2013;8:e80633.

27 Hess LM, Raebel MA, Conner DA, et al. Measurement of adherence in pharmacy administrative databases: a proposal for standard definitions and preferred measures. Ann Pharmacother 2006;40:1280-8.

28 Malo S, Aguilar-Palacio I, Feja C, et al. Different approaches to the assessment of adherence and persistence with cardiovascular-disease preventive medications. Curr Med Res Opin 2017;33:1329-36.

29 Caetano PA, Lam JMC, Morgan SG. Toward a standard definition and measurement of persistence with drug therapy: examples from research on statin and antihypertensive utilization. Clin Ther 2006;28:1411-24.

30 Jackevicius CA, Li P, Tu JV. Prevalence, predictors, and outcomes of primary nonadherence after acute myocardial infarction. Circulation 2008;117:1028-36 